Supplement of Biogeosciences Discuss., 12, 13159-13192, 2015

http://www.biogeosciences-discuss.net/12/13159/2015/

doi:10.5194/bgd-12-13159-2015-supplement

(C) Author(s) 2015. CC Attribution 3.0 License.

(c) (1)

Supplement of

\title{
Spatial and temporal trends in summertime climate and water quality indicators in the coastal embayments of Buzzards Bay, Massachusetts
}

\section{J. E. Rheuban et al.}

Correspondence to: J. E. Rheuban (jrheuban@whoi.edu)

The copyright of individual parts of the supplement might differ from the CC-BY 3.0 licence. 


\section{Supplement}

An example of the T, TN, and Chla time series from one embayment (Sippican Harbor, \#11) is given in Fig. S1. In this example, each point reflects the average of 7 stations, with $\sim 40$ measurements per year. While there is considerable interannual variability, linear regressions reveal a positive slope over time in all three parameters, though a statistically significant increasing trend is only observed for $\mathrm{T}$ and Chl. As seen in Figure 3, the pattern exhibited in Sippican Harbor of statistically significant increasing trends in $\mathrm{T}$ and $\mathrm{Chl}$ was common around Buzzards Bay.
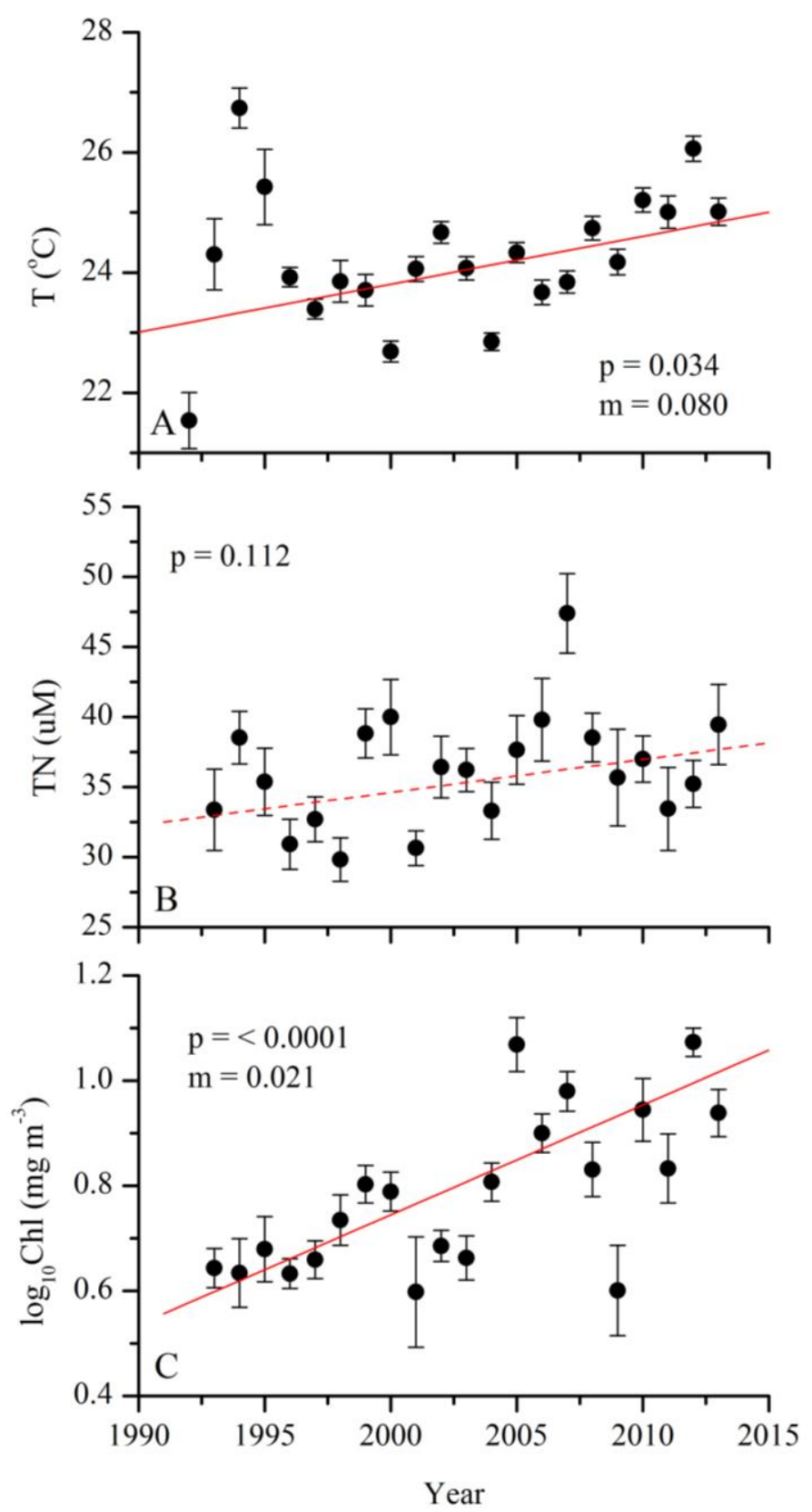

Fig. S1. July/August averages of (A) temperature ( $T$ ), (B) total nitrogen (TN), and (C) chlorophyll (Chla) from Sippican Harbor (\#9). Lines are long term trends fitted using linear regression, solid lines indicate statistical significant at the $\alpha=0.05$ level. Error bars are standard error. 
Table S1. Mean, standard deviation (SD), and total number of samples ( $n$ ) of physical parameters across embayments.

\begin{tabular}{|c|c|c|c|c|c|c|c|c|c|c|c|c|c|}
\hline \multirow[b]{2}{*}{ Embayment Name } & \multirow[b]{2}{*}{ \# } & \multicolumn{3}{|c|}{$\mathrm{T}$} & \multicolumn{3}{|c|}{ Salt } & \multicolumn{3}{|c|}{$\mathrm{DO}$} & \multicolumn{3}{|c|}{ DOsat } \\
\hline & & $\operatorname{deg} C$ & SD & $\mathrm{n}$ & $\mathrm{ppt}$ & $\mathrm{SD}$ & $\mathrm{n}$ & $\mathrm{mg} / \mathrm{L}$ & SD & $\mathrm{n}$ & $\begin{array}{c}\% \\
\text { sat }\end{array}$ & $\mathrm{SD}$ & $\mathrm{n}$ \\
\hline Westport River & 1 & 22.6 & 2.4 & 3088 & 27.0 & 7.8 & 3130 & 6.1 & 1.3 & 2287 & 0.82 & 0.18 & 2268 \\
\hline Slocums River & 2 & 22.9 & 2.4 & 1344 & 25.3 & 8.8 & 1351 & 5.9 & 1.1 & 905 & 0.81 & 0.13 & 736 \\
\hline Apponagansett Bay & 3 & 23.1 & 2.2 & 1629 & 28.7 & 6.9 & 1700 & 5.8 & 1.1 & 864 & 0.81 & 0.15 & 859 \\
\hline Clarks Cove & 4 & 23.4 & 1.7 & 431 & 31.0 & 1.5 & 440 & 5.8 & 1.3 & 142 & 0.82 & 0.18 & 140 \\
\hline $\begin{array}{c}\text { New Bedford } \\
\text { Harbor }\end{array}$ & 5 & 23.2 & 1.9 & 1324 & 27.6 & 8.2 & 1346 & 5.9 & 1.5 & 652 & 0.81 & 0.21 & 647 \\
\hline Nasketucket Bay & 6 & 23.1 & 2.0 & 777 & 27.9 & 6.4 & 827 & 5.3 & 1.5 & 518 & 0.73 & 0.22 & 516 \\
\hline Mattapoisett Harbor & 7 & 23.1 & 2.0 & 1660 & 29.1 & 3.9 & 1933 & 5.5 & 1.3 & 1007 & 0.76 & 0.18 & 1006 \\
\hline Aucoot Cove & 8 & 23.2 & 1.8 & 903 & 30.5 & 2.0 & 916 & 5.2 & 1.7 & 438 & 0.73 & 0.23 & 438 \\
\hline Sippican Harbor & 9 & 24.2 & 2.0 & 1197 & 30.0 & 2.7 & 1230 & 5.3 & 1.1 & 713 & 0.75 & 0.15 & 707 \\
\hline Wareham River & 10 & 24.1 & 1.8 & 3161 & 22.8 & 6.7 & 3434 & 5.8 & 1.0 & 1503 & 0.79 & 0.13 & 1501 \\
\hline Onset Bay & 11 & 21.3 & 2.1 & 1746 & 30.0 & 1.9 & 1940 & 7.2 & 1.1 & 1144 & 0.96 & 0.14 & 1135 \\
\hline Buttermilk Bay & 12 & 22.1 & 2.3 & 946 & 28.8 & 1.8 & 989 & 6.3 & 0.9 & 313 & 0.86 & 0.12 & 313 \\
\hline $\begin{array}{c}\text { Eel Pond/Phinneys } \\
\text { Harbor } \\
\text { Red Brook }\end{array}$ & 13 & 22.9 & 2.0 & 820 & 29.1 & 2.6 & 923 & 6.3 & 1.2 & 440 & 0.86 & 0.16 & 438 \\
\hline $\begin{array}{c}\text { Harbor/Pocasset } \\
\text { River }\end{array}$ & 14 & 24.0 & 2.0 & 2007 & 29.6 & 2.0 & 2373 & 5.8 & 1.0 & 1193 & 0.82 & 0.14 & 1187 \\
\hline Megansett Harbor & 15 & 23.4 & 1.8 & 2138 & 29.0 & 2.4 & 2146 & 6.2 & 0.9 & 1520 & 0.86 & 0.13 & 1514 \\
\hline $\begin{array}{l}\text { West Falmouth } \\
\text { Harbor }\end{array}$ & 16 & 22.5 & 1.9 & 1006 & 28.0 & 3.8 & 1685 & 6.1 & 1.1 & 968 & 0.83 & 0.15 & 959 \\
\hline Quissett Harbor & 17 & 22.5 & 1.6 & 706 & 31.0 & 1.5 & 706 & 6.5 & 1.0 & 561 & 0.89 & 0.14 & 560 \\
\hline
\end{tabular}


Table S2. Mean, standard deviation (SD), and total number of samples ( $n$ ) of inorganic nutrient parameters across embayments.

\begin{tabular}{c|cccccccccc}
\hline Embayment Name & \multicolumn{4}{|c}{$\mathrm{NO}_{3}+\mathrm{NO}_{2}$} & \multicolumn{3}{c}{$\mathrm{NH}_{4}$} & \multicolumn{3}{c}{$\mathrm{PO}_{4}$} \\
& & $\mathrm{uM}$ & $\mathrm{SD}$ & $\mathrm{n}$ & $\mathrm{uM}$ & $\mathrm{SD}$ & $\mathrm{n}$ & $\mathrm{uM}$ & $\mathrm{SD}$ & $\mathrm{n}$ \\
\hline \hline Westport River & 1 & 3.4 & 9.0 & 1041 & 3.2 & 4.1 & 1042 & 1.1 & 0.8 & 1043 \\
Slocums River & 2 & 4.8 & 10.0 & 409 & 2.3 & 2.7 & 409 & 1.1 & 0.6 & 409 \\
Apponagansett Bay & 3 & 7.2 & 20.0 & 499 & 2.2 & 2.8 & 499 & 1.8 & 0.8 & 499 \\
Clarks Cove & 4 & 0.4 & 0.5 & 250 & 1.0 & 1.1 & 250 & 1.9 & 1.3 & 250 \\
New Bedford Harbor & 5 & 7.4 & 14.6 & 356 & 3.7 & 4.2 & 359 & 2.1 & 1.0 & 357 \\
Nasketucket Bay & 6 & 2.4 & 5.9 & 319 & 1.7 & 1.8 & 319 & 0.9 & 0.4 & 321 \\
Mattapoisett Harbor & 7 & 1.2 & 2.5 & 665 & 1.2 & 1.4 & 665 & 0.8 & 0.4 & 665 \\
Aucoot Cove & 8 & 0.6 & 1.2 & 394 & 1.3 & 1.3 & 394 & 1.0 & 0.5 & 394 \\
Sippican Harbor & 9 & 0.4 & 0.7 & 572 & 1.1 & 1.3 & 573 & 1.0 & 1.1 & 573 \\
Wareham River & 10 & 0.5 & 0.6 & 1197 & 1.8 & 2.4 & 1197 & 1.0 & 0.4 & 1197 \\
Onset Bay & 11 & 0.3 & 0.4 & 551 & 1.1 & 1.0 & 550 & 0.5 & 0.2 & 550 \\
Buttermilk Bay & 12 & 0.6 & 1.4 & 460 & 1.3 & 1.5 & 462 & 0.6 & 0.4 & 461 \\
Eel Pond/Phinneys Harbor & 13 & 0.7 & 1.4 & 430 & 1.1 & 1.2 & 433 & 0.6 & 0.4 & 433 \\
Red Brook Harbor/Pocasset River & 14 & 0.5 & 0.9 & 696 & 1.2 & 1.5 & 689 & 0.7 & 0.6 & 688 \\
Megansett Harbor & 15 & 0.6 & 1.0 & 547 & 0.9 & 0.8 & 548 & 0.5 & 0.5 & 548 \\
West Falmouth Harbor & 16 & 2.1 & 5.5 & 1610 & 1.6 & 1.9 & 1610 & 0.6 & 0.5 & 1616 \\
Quissett Harbor & 17 & 0.5 & 0.6 & 166 & 1.5 & 1.5 & 166 & 0.7 & 0.2 & 166 \\
\hline
\end{tabular}


Table S2. Mean, standard deviation (SD), and total number of samples (n) of organic nutrient parameters across embayments.

\begin{tabular}{|c|c|c|c|c|c|c|c|c|c|c|c|c|c|}
\hline \multirow[b]{2}{*}{ Embayment Name } & \multirow[b]{2}{*}{$\#$} & \multicolumn{3}{|c|}{ PON } & \multicolumn{3}{|c|}{ POC } & \multicolumn{3}{|c|}{$\mathrm{DON}$} & \multicolumn{3}{|c|}{$*$ ChlA } \\
\hline & & $\mathrm{uM}$ & $\mathrm{SD}$ & $\mathrm{n}$ & $\mathrm{uM}$ & $\mathrm{SD}$ & $\mathrm{n}$ & $\mathrm{uM}$ & $\mathrm{SD}$ & $\mathrm{n}$ & $\begin{array}{l}\mathrm{mg} \\
\mathrm{m} 3\end{array}$ & $\mathrm{SD}$ & $\mathrm{n}$ \\
\hline Westport River & 1 & 10.9 & 8.1 & 1033 & 77.1 & 57.6 & 1032 & 38.8 & 24.4 & 1037 & 4.1 & 2.3 & 1019 \\
\hline Slocums River & 2 & 17.5 & 11.9 & 398 & 134.5 & 98.5 & 397 & 28.1 & 15.0 & 409 & 6.5 & 2.6 & 407 \\
\hline $\begin{array}{l}\text { Apponagansett } \\
\text { Bay }\end{array}$ & 3 & 14.5 & 16.0 & 484 & 110.0 & 141.1 & 484 & 21.5 & 10.4 & 497 & 6.2 & 2.2 & 536 \\
\hline Clarks Cove & 4 & 8.8 & 9.5 & 242 & 67.1 & 72.5 & 242 & 23.1 & 14.9 & 248 & 3.8 & 2.0 & 289 \\
\hline $\begin{array}{c}\text { New Bedford } \\
\text { Harbor }\end{array}$ & 5 & 16.5 & 14.8 & 347 & 118.7 & 101.2 & 347 & 27.9 & 17.4 & 355 & 8.3 & 2.5 & 298 \\
\hline Nasketucket Bay & 6 & 12.1 & 11.1 & 307 & 97.1 & 121.2 & 307 & 25.8 & 14.3 & 318 & 5.2 & 1.8 & 251 \\
\hline $\begin{array}{l}\text { Mattapoisett } \\
\text { Harbor }\end{array}$ & 7 & 10.4 & 8.0 & 645 & 79.0 & 64.9 & 645 & 19.9 & 10.6 & 664 & 4.2 & 2.0 & 783 \\
\hline Aucoot Cove & 8 & 8.3 & 5.1 & 386 & 64.5 & 44.1 & 387 & 18.6 & 8.3 & 394 & 3.3 & 1.9 & 552 \\
\hline Sippican Harbor & 9 & 12.3 & 6.2 & 561 & 95.4 & 54.2 & 561 & 22.3 & 9.1 & 571 & 6.1 & 2.0 & 568 \\
\hline Wareham River & 10 & 14.3 & 8.8 & 1167 & 112.1 & 98.3 & 1167 & 23.0 & 13.7 & 1192 & 7.5 & 2.1 & 1612 \\
\hline Onset Bay & 11 & 7.9 & 5.2 & 541 & 55.4 & 30.1 & 542 & 17.8 & 7.2 & 549 & 4.1 & 1.6 & 547 \\
\hline Buttermilk Bay & 12 & 11.2 & 8.5 & 454 & 75.3 & 48.3 & 454 & 19.0 & 7.9 & 459 & 5.2 & 1.9 & 456 \\
\hline $\begin{array}{l}\text { Eel Pond/Phinneys } \\
\text { Harbor }\end{array}$ & 13 & 8.9 & 3.4 & 422 & 64.1 & 27.7 & 422 & 18.8 & 8.4 & 429 & 4.6 & 2.0 & 423 \\
\hline $\begin{array}{c}\text { Red Brook } \\
\text { Harbor/Pocasset } \\
\text { River }\end{array}$ & 14 & 10.2 & 5.8 & 687 & 78.0 & 50.9 & 686 & 22.0 & 11.3 & 688 & 4.8 & 2.1 & 691 \\
\hline Megansett Harbor & 15 & 9.7 & 7.9 & 533 & 69.1 & 52.0 & 533 & 19.1 & 10.7 & 546 & 4.5 & 2.0 & 545 \\
\hline $\begin{array}{c}\text { West Falmouth } \\
\text { Harbor }\end{array}$ & 16 & 10.9 & 7.2 & 1590 & 60.0 & 29.6 & 654 & 22.4 & 12.5 & 1603 & 3.2 & 2.1 & 616 \\
\hline Quissett Harbor & 17 & 5.6 & 2.5 & 163 & 43.2 & 19.5 & 163 & 16.0 & 5.0 & 164 & 2.3 & 1.8 & 165 \\
\hline
\end{tabular}

*indicates geometric mean and standard deviation 

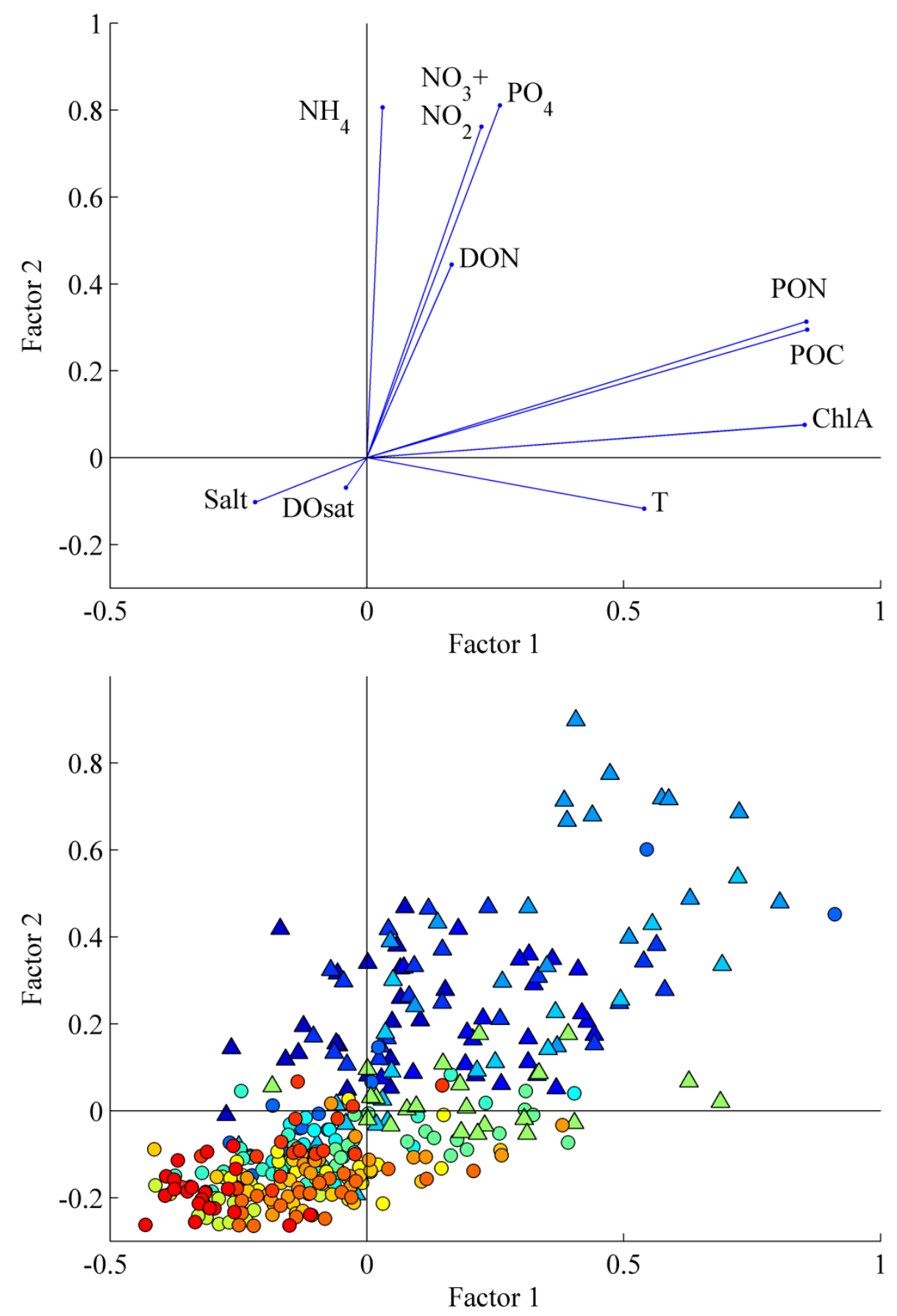

Fig. S2 Factor loadings (top panel) and factor scores (bottom panel) showing factor scores for each year, from each embayment. Colors correspond to embayments in Figure 1. Triangles are embayments designated 'river-fed', and circles are embayments designated 'groundwater-fed'. 